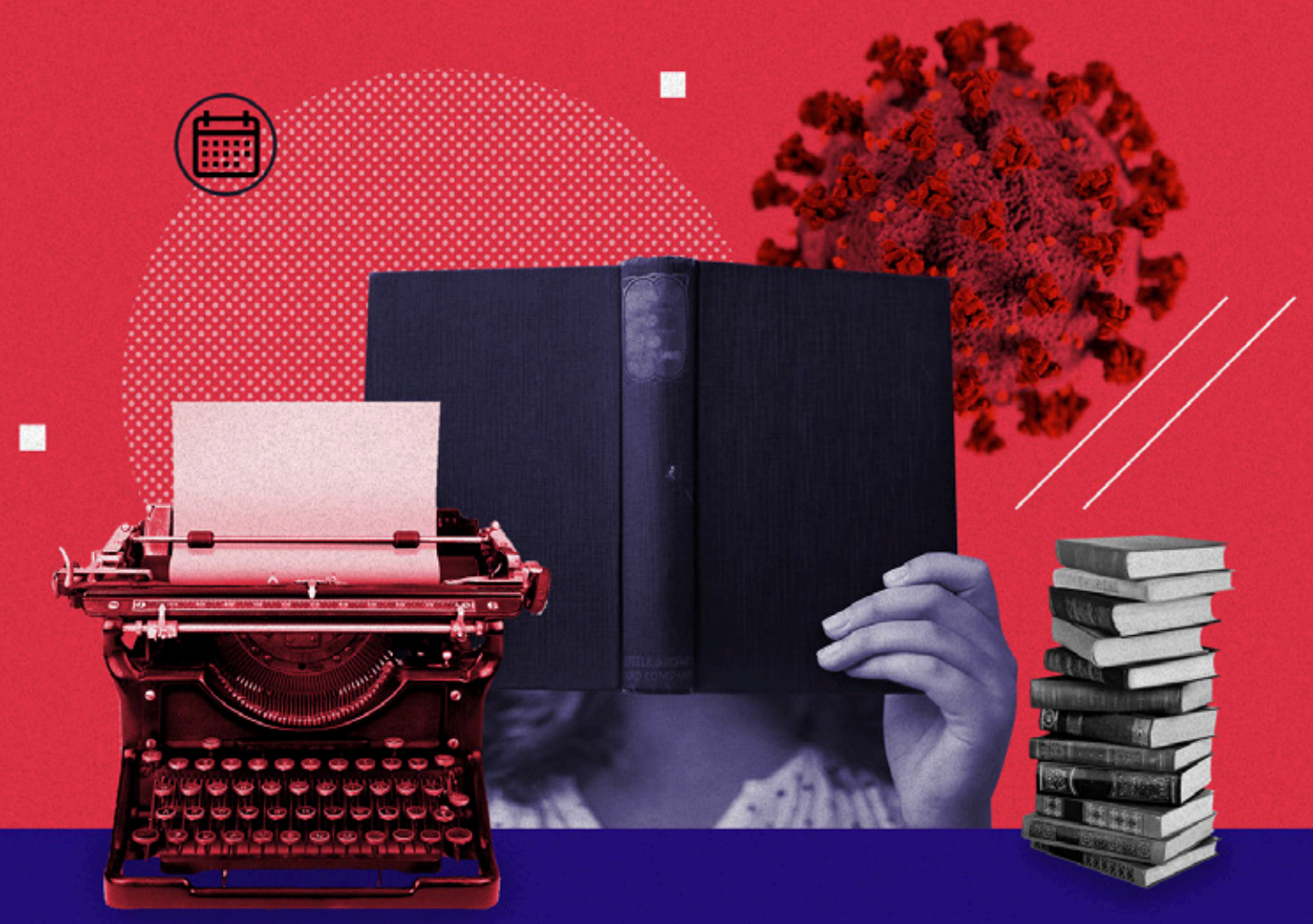

\title{
Leer y escribir en tiempos de pandemia
}

READING AND WRITING DURING PANDEMIC TIMES

LER E ESCREVER EM TEMPOS DE PANDEMIA

Karla Irene Martínez Méndez ${ }^{1}$ 
Citar artículo como:

Martínez-Méndez, K.I (2021). Leer y escribir en tiempos de pandemia. Educación y Ciudad, n. 41, pp. 7186. https://doi.org/10.36737/01230425.n41.2021.2530

Fecha de recepción: 9 de abril de 2021

Fecha de aprobación: 6 de julio de 2021

\section{Resumen}

La presente investigación se enmarca en el paradigma cualitativo y tiene como objetivo conocer cómo han llevado a cabo su práctica docente nueve maestros de educación primaria, estudiantes del programa de maestría en educación primaria de la Benemérita y Centenaria Escuela Normal del Estado de San Luis Potosí, México, haciendo énfasis en la enseñanza de la lectura y escritura a través de la virtualidad. Los resultados muestran que los docentes han mantenido comunicación con sus alumnos a través de WhatsApp, plataformas virtuales y sesiones sincrónicas. Respecto a las estrategias de enseñanza-aprendizaje en relación con la lectura y escritura, se mencionan los foros de lectura, escritura y redacción de textos diversos.

Palabras clave: Lectura; escritura; práctica docente; estrategias de enseñanza; educación virtual.

\section{Abstract}

This paper is framed under the qualitative paradigm and its objective is to review how nine elementary school teachers, students of the master's program in primary education of La Benemérita y Centenaria Escuela Normal of the State of San Luis Potosí, México, have carried on their professional practice, putting emphasis in the teaching of reading and writing skills through virtual technology means. Results show how teachers have kept communication with their students through means like WhatsApp, virtual platforms, and synchronous sessions. Regarding the teaching-learning strategies that are related to reading and writing, reading, writing, and drafting forums about diverse texts are considered.

Keywords: Reading; writing; teaching practice; teaching strategies; virtual learning.

\section{Resumo}

Esta pesquisa está enquadrada no paradigma qualitativo e tem como objetivo conhecer como nove professores da educação primária vêm realizando a sua prática docente, alunos do Programa de Mestrado em Educação Básica da Benemérita e Centenaria Escola Normal do Estado de San Luis Potosí, México, com ênfase no ensino da leitura e da escrita por meio da virtualidade. Os resultados mostram que os professores mantêm a comunicação com seus alunos pelo WhatsApp, plataformas virtuais e sessões síncronas. No que se refere às estratégias de ensino-aprendizagem em relação à leitura e escrita, são mencionados os fóruns para leitura e redação e para escrita de textos diversos.

Palavras-chave: Leitura; escrita; prática docente; estratégias de ensino: educação virtual. 


\section{Introducción}

工 os servicios de salud mundial mantienen su atención ante la alerta de pandemia emitida por la Organización Mundial de la Salud (OMS) originada por el coronavirus y que se ha identificado como COVID-19 (Expansión política, 2021). Según datos de la OMS, la pandemia por COVID-19 afectó a 216 países en el mundo y golpeó a todos los países de las Américas en 2020. En México, y según datos de la Secretaría de Salud al día 06 de enero del 2021, se registraron 11.271 contagios nuevos y 1.065 defunciones, por lo que la cifra de casos acumulados ascendió a 1.466590 , mientras que el total de las muertes se elevó a 128.822 (INFOBAE, 2021).

Ante esto, la Secretaría de Educación Pública (SEP) de México, anunció que las clases se mantendrían suspendidas desde el viernes 20 de marzo y hasta nuevo aviso, intentando con esto, proteger a niños y niñas, y así, prevenir más contagios en el país. La medida de suspensión de clases aplicaba para todo el sector educativo nacional; esto obligó a docentes y estudiantes a desarrollar actividades educativas bajo la modalidad virtual, haciendo uso de plataformas u otras herramientas tecnológicas con el fin de concluir el ciclo escolar 2019-2020.

Ante esto, México activó el semáforo epidemiológico compuesto por cuatro colores, rojo, naranja, amarillo y verde; este último, indica el riesgo epidemiológico población como bajo. De acuerdo con lo anterior, el secretario de Educación informó que el regreso a cla-

Doctora en Ciencias Sociales de la Benemérita y Centenaria Escuela Normal del Estado (BECENE) de San Luis Potosí, México. ORCID: https://orcid.org/0000-0001-7063-2642 Correo electrónico: kmartinezmendez@hotmail.com ses se realizará cuando el semáforo de control de la pandemia se encuentre en color verde. Al momento de escribir estas líneas, marzo del 2021, la entidad a la que pertenece la autora se encuentra en color naranja, indicando un riesgo epidemiológico alto, solo debajo del rojo que indica riesgo máximo. Por ello, se mantienen las clases bajo una modalidad en línea (Acuerdo número 02/03/20).

Luego de semanas de aislamiento por el coronavirus, el 20 de abril del 2020 el gobierno mexicano en conjunto con la SEP y las autoridades de la Salud de la nación, iniciaron el programa "Aprende en casa I" como estrategia educativa virtual para cumplir el calendario escolar. Este programa, permitiría que casi 30 millones de estudiantes reanudaran sus actividades escolares por televisión abierta. La base de este programa se encuentra en el Libro de Texto Gratuito al que tienen acceso las niñas y niños del país (SEP, 2020a).

Por su parte, el Subsecretario de Educación Básica, indicaba que el propósito de esta estrategia era facilitar la continuidad de estudiar en casa y crear hábitos para fortalecer la educación a distancia, al tiempo de divertirse y aprender en familia. El programa se basa en los aprendizajes fundamentales de cada grado escolar y garantiza la continuidad de la educación sin ejercer una presión adicional para las familias (SEP, 2020b).

Sumado a lo anterior, la SEP a través de la Subsecretaria de Educación Básica, creó un sitio web en 
apoyo al programa Aprende en Casa I (SEP, 2020c). Este sitio, permitiría tener acceso a archivos electrónicos y páginas temáticas, así como materiales educativos en versión digital (videos, audios, documentos, guías de estudio, infografías, Formato de Intercambio de Gráficos (GIF por sus siglas en inglés)) folletos, consejos para leer mejor y trabajar en equipo, entre otros (SEP, 2020c).

A pesar de los esfuerzos de diversas instituciones científicas en el mundo, el nivel de la pandemia de COVID-19 alcanzó dimensiones inimaginables (Banco Interamericano de Desarrollo, 2020). Este escenario obligó a las autoridades mexicanas a mantener suspendidas la asistencia a las instituciones educativas e iniciar el Ciclo Escolar 2020-2021 desde casa. En este marco, se anunció el inicio del programa "Aprende en casa II" (SEP, 2020d). Además, se determinó que también estaría disponible en Internet, por medio de un canal en YouTube.

Esta investigación tiene como objetivo conocer cómo han llevado a cabo su práctica docente 9 maestros de educación primaria poniendo énfasis en la enseñanza de la lectura y escritura a través de la virtualidad.

\section{Desarrollo}

Los docentes que participan en este estudio son 9. Todos son maestros en el nivel primaria, ocho laboran en escuelas primarias de San Luis Potosí y solo una maestra en el estado de Guanajuato, México. Todos son estudiantes del programa de maestría en educación primaria de la Benemérita y Centenaria Escuela Normal del Estado (BECENE) de San Luis Potosí.
En caso de la maestra Fernanda, su grupo de primero de primaria está conformado por un total de 28 alumnos en esta escuela primaria de tipo pública. Según la docente, hasta el momento únicamente ha tenido contacto con un total de 20 de familia ; los otros 8 no responden sus mensajes o no envían las actividades de sus hijos.

El maestro Carlos, labora en una escuela primaria de tipo pública, es responsable del $2^{\circ}$ grado conformado por 33 estudiantes. Carlos los define como alumnos creativos, dinámicos, de aprendizaje activo y práctico.

La maestra Juana es responsable del grupo de $2^{\circ}$ grado, con 20 alumnos, en una primaria de tipo pública. Está ubicada en un medio rural y es una zona que cuenta con todos los servicios básicos como agua, luz, teléfono y algunas zonas internet.

En el caso de la maestra Dulce, atiende 3er. año de primaria. Es una escuela de tipo privada cercana al centro de la ciudad. Ella refiere que son 32 alumnos en total. Todos los días se revisan 4 materias incluyendo español y matemáticas, además de artes, inglés y educación física.

El grupo de la maestra Lucero es de $6^{\circ}$. Grado y consta de 22 alumnos. Es una institución pública ubicada en la capital potosina.

El grupo de la maestra Mariana cursa el $2^{\circ}$. grado y está conformado por 27 alumnos. Afirma que hay niños que aún no alcanzan el proceso de lectoescritura.

La maestra Minerva atiende el grupo de 1er. Grado; cuenta con un total de 12 alumnos. La escuela es pú- 
blica. La maestra afirma que se encuentra en comunicación constante con 10 de los padres de familia que son el vínculo directo con los niños, mientras que con 2 de ellos no se ha podido establecer comunicación.

El maestro Nicolás labora en una escuela pública en el medio rural; tiene 1er. grado. El grupo se encuentra conformado por 17 alumnos; según el docente, la mayoría son participativos y cuentan con el apoyo de los papás.

El grupo de la maestra Sara es de $2^{\circ}$. grado, en escuela primaria pública, en el turno vespertino. Está conformado por 20 alumnos.

Abandonar las aulas presenciales a todos nos tomó por sorpresa. De un momento a otro, los diversos actores educativos debieron adaptarse (Mendoza, 2020) a un modelo educativo en línea, virtual, a distancia; a dejar los edificios por las plataformas virtuales y emplear aquellas herramientas tecnológicas que permitan continuar con el proceso educativo en la medida de lo posible.

Los estudiantes reconocieron la extrañeza de la situación y la necesidad de convivencia en el aula. Expresaron extrañar a sus compañeros y a su maestro. Los docentes por su parte se enfrentaron a un duro reto, impartir sus materias detrás de una cámara, dar seguimiento a su grupo a través de las plataformas $\mathrm{u}$ otro medio para dar continuidad a su labor como docente. La práctica docente se transformó para adaptarse a las necesidades.

La práctica docente de acuerdo con De Lella (1999 en García, Loredo y Carranza, 2008), se entiende como la acción que el profesor desarrolla en el aula, especialmente referida al proceso de enseñar, y se distingue de la práctica institucional global y la práctica social del docente. De acuerdo con García, Loredo y Carranza (2008), la práctica docente se concibe como el conjunto de situaciones dentro del aula, que configuran el quehacer del profesor y de los alumnos, en función de determinados objetivos de formación circunscritos al conjunto de actuaciones que inciden directamente sobre el aprendizaje de los alumnos. A diferencia de la práctica docente, Zabala (1995) señala que la práctica educativa obedece a múltiples determinantes, pues tiene justificación en parámetros institucionales, organizativos, tradiciones metodológicas, posibilidades reales de los profesores, de los medios y las condiciones físicas existentes, entre otros.

Por otro lado, la educación a distancia puede entenderse, como el proceso de enseñanza-aprendizaje, que, a diferencia de la educación presencial, emplea medios digitales para la interactividad síncrona o asíncrona (Martínez, 2008). Esta educación virtual puede conceptualizarse como una forma de enseñanza en la que los estudiantes no necesariamente tienen que asistir de forma física al centro escolar, y pueden avanzar a su propio ritmo de aprendizaje de acuerdo con su capacidad y disponibilidad de tiempo. El contacto personal entre maestro y alumno puede ser poco o nulo, mientras que en otros se requiere asistir con determinada periodicidad (González, 2005).

Peters (2002) afirma que la educación a distancia ha significado una ruptura que ocasionó:

"el cambio de los espacios reales de aprendizaje por los espacios virtuales. Este cambio significa que los maestros y estudiantes ahora se ven expuestos a una situación que tiene una estructura completamente 
diferente y que ofrece una variedad de oportunidades nuevas" (p.134).

Entre las ventajas de la modalidad virtual, Pedraza (2003, en Ralón, Vieta y Vásquez, 2003) considera que los entornos virtuales amplían el acceso a la educación, promueven el aprendizaje colaborativo y el trabajo en grupo, promueven el aprendizaje activo, crean comunidades de aprendizaje, se centra en el estudiante y hace que los roles tradicionales del proceso de enseñanza-aprendizaje sean concebidos de manera más estructurada y organizada.

\section{Metodología}

Este estudio se inscribe dentro del paradigma cualitativo, de tipo exploratorio-descriptivo. Este tipo de investigación se caracteriza por buscar, comprender y describir algún suceso externo de forma no fragmentada, en tanto el investigador se involucra en la realidad. Rodríguez, Gil y García (2006) afirman: "los investigadores cualitativos estudian la realidad en su contexto natural, tal como sucede, intentando sacar sentido de, o interpretar, los fenómenos de acuerdo con los significados que tienen para las personas implicadas" (p. 72).

Los maestros que cursaban el primer semestre de la Maestría en educación primaria, se les invitó a participar en el presente estudio, exponiéndoles los objetivos de la investigación y solicitándoles su consentimiento para incluirlos. Los participantes de esta investigación son siete maestras y dos maestros; todos se encuentran frente a grupo de nivel primaria y son estudiantes del programa de maestría en educación primaria de la BECENE.

Para la recolección de datos se solicitó un ensayo acerca de su experiencia con su grupo de primaria en este periodo de pandemia respecto a:

a. El contexto de trabajo

b. Las formas de contacto y comunicación con el grupo

c. La forma en la que conduce su grupo respecto a la lectura y escritura bajo las condiciones de cuarentena que experimentamos.

Después de recibir los ensayos de cada participante, se procedió a organizar la información en tablas según los apartados que se solicitaron. Cada temática se consideró una categoría de análisis. Estos datos fueron organizados de acuerdo con similitudes en contenido, y se analizaron y contrastaron con la teoría para presentar los resultados.

\section{Resultados y discusión}

\section{Formas de contacto y comunicación con el grupo}

Entre los retos de los maestros para lleva a cabo sus actividades docentes se encuentra la comunicación con sus alumnos. De acuerdo con datos del Instituto Nacional de Estadística y Geografía-INEGI (2018) en México, solo un 44,3\% del total de los hogares cuentan con computadora (Tabla 1). 
Tabla 1. Encuesta Nacional sobre Disponibilidad y Uso de TIC en Hogares en México.

\begin{tabular}{|c|c|c|c|c|}
\hline Indicadores sobre Disponibilidad y Uso de TIC & 2016 & 2017 & 2018 & 2019 \\
\hline $\begin{array}{l}\text { Hogares con computadora como proporción del total } \\
\text { de hogares }\end{array}$ & 45,6 & 45,4 & 44,9 & 44,3 \\
\hline $\begin{array}{l}\text { Hogares con conexión a Internet como proporción del } \\
\text { total de hogares }\end{array}$ & 47,0 & 50,9 & 52,9 & 56,4 \\
\hline $\begin{array}{l}\text { Hogares con televisión como proporción del total de } \\
\text { hogares }\end{array}$ & 93,1 & 93,2 & 92,9 & 92,5 \\
\hline $\begin{array}{l}\text { Usuarios de computadora como proporción de la } \\
\text { población de seis años o más de edad }\end{array}$ & 47,0 & 45,3 & 45,0 & 43,0 \\
\hline $\begin{array}{l}\text { Usuarios de computadora que la usan como herramienta } \\
\text { de apoyo escolar como proporción del total de usuarios } \\
\text { de computadora }\end{array}$ & 52,2 & 46,8 & 46,7 & 44,6 \\
\hline
\end{tabular}

Fuente: INEGI De 2015-2018: Encuesta Nacional sobre Disponibilidad y Uso de TIC en Hogares, ENDUTIH.

La educación a distancia surge como una estrategia para hacer llegar la educación a todo aquel que la necesita (Bates, 1995 en Navarrete y Manzanilla, 2017) y es implementada en este periodo de pandemia para hacer frente a la necesidad de que los niños se queden en casa, y así, prevenir los contagios. Es importante considerar que uno de los grandes retos de la educación a distancia es el establecimiento de la comunicación entre el estudiante y su maestro.

Según Bates (1995 en Navarrete y Manzanilla, 2017), en la educación a distancia se requiere de un elemento mediador entre el docente y el estudiante; generalmente este mediador ha sido una tecnología que ha ido variando de acuerdo con el avance de las Tecnologías de la Información y Comunicación (TICs). Históricamente se empleaba el correo convencional, que establecía una relación entre el maestro y sus estudiantes, posteriormente, se han ido empleando otros medios y tecnologías de menor costo, mayor acceso y de manejo más amigable que permiten poner en práctica la educación a distancia con mayor frecuencia.

Respecto a los docentes de primaria que participan, afirman que el principal medio de comunicación con sus estudiantes es a través de WhatsApp, videollamadas y llamadas telefónicas a alumnos. En otros casos, y previa comunicación con los padres de familia, no fue posible el uso de plataformas virtuales por la falta de familiaridad con el uso y manejo de éstas, como lo prueban los testimonios de la maestra Sara:

"Creé un grupo de WhatsApp como primera vía de comunicación con los padres de familia y tutores, para avisos y compartir las actividades de manera 
semanal, que serían revisadas de forma diaria para dar retroalimentación".

"No era viable utilizar Classroom, pues los padres de familia consideraban que la plataforma era de difícil uso, por lo que se optó por enviar las evidencias a través de WhatsApp y el correo institucional”.

Además, cabe recordar que para acceder y mantener el servicio de internet es necesario que los padres de familia destinen parte de su presupuesto para tal fin, pero esto no ha sido posible en todos los hogares. El ingreso es escaso en algunos, en otros -y debido a la pandemia- algunos padres de familia perdieron su empleo, lo que dificulta mantener la conexión a internet, como lo evidencia este otro testimonio de la maestra Minerva:

"Prácticamente la mayoría [de los padres de familia] había indicado que no contaban con internet de banda ancha y se les dificultaba "meterle" saldo para tener datos, pues algunos padres de familia habían perdido su empleo".

Según Loyola (2017), en algún momento se vio a la educación a distancia como la opción que podría comenzar a resolver algunos de los problemas educativos del nuevo siglo, sin embargo, la falta de acceso a internet continúa limitando la apertura de la educación a distancia, como lo dice la profesora Mariana:

"El medio de comunicación para las actividades es por la aplicación de WhatsApp, solo una mamá no cuenta con esta conexión y lo que se hace es dar seguimiento por medio de llamadas telefónicas".

Para lograr acceder a la educación a distancia, es indispensable contar con señal de internet de calidad, pero aún hoy en día, existen espacios sin ac- ceso como las zonas distantes a la urbe (Balmaceda, 2020 en Beltramino, 2020).

Según Huesca (2015), la educación a distancia no influye en las zonas marginadas, pues parece que la conectividad sólo está disponible en zonas urbanas de clase media, y específicamente, en espacios en donde se cuenta ampliamente con los servicios comerciales de telecomunicaciones, e incluso en estos espacios, se puede cuestionar la eficiencia. La amplia gama de proveedores no ha sido suficiente, la mayoría se concentra en los espacios urbanos, sin lograr que los niños de las comunidades alejadas accedan al servicio de internet y, por lo tanto, a la educación (Huesca, 2015).

Para desarrollar las actividades educativas, los docentes iniciaron el curso estableciendo contacto con los padres; algunos realizaron una encuesta a través de "formularios Google" con el objetivo de conocer los recursos tecnológicos con los que se contaban. De acuerdo con las respuestas, los docentes diseñaron estrategias de desarrollo y seguimiento de las actividades educativas, como lo dice Minerva:

\begin{abstract}
"Cuando se inició el ciclo escolar, se hizo una encuesta realizada a padres de familia a través de "formularios Google", se les preguntaba los recursos con que se contaba. Más de la mitad de los padres de familia indicaban que sí contaban con televisión, por lo que se inició trabajando con la programación de "Aprende en Casa" y a través de WhatsApp se enviaban las evidencias, esto, porque aún no se contaban con los libros completos"
\end{abstract}

Cuando los docentes identifican que en los hogares se tiene acceso al uso de ciertas TICs, expresan que realizan seguimiento a sus estudiantes a través 
de los medios más comunes: WhatsApp, llamadas y videollamadas frecuentes, e incluso, a través del uso de plataformas como Google Meet y Classroom (Barrón, 2020), según los testimonios de Juana y Fernanda, maestras que fueron parte de la investigación:

"Es mediante un grupo de WhatsApp con padres de familia en donde cada semana se manda el plan a trabajar por día y el material a utilizar como cuadernillos de trabajo, videos, audios o recortables. Los viernes se reciben evidencias de los trabajos de clase, así como imágenes o videos para la revisión y retroalimentación de cada uno de ellos. Mediante el grupo se organiza por día las videollamadas para estar con los niños cada semana, y también se envían materiales complementarios como bibliotecas virtuales, ruletas, audio cuentos, canciones de multiplicaciones, leyendas o imágenes que pueden ayudarles en la realización de sus actividades".

"El contacto con los padres de familia del grupo se desarrolla a partir de WhatsApp para mandar las actividades, una vez a la semana hay clase grupal a través de la plataforma de Google Meet para abordar los temas y dudas que surgen".

Para dar continuidad a las actividades educativas algunos docentes han optado por realizar sesiones sincrónicas con sus estudiantes, considerando que existen contenidos "complejos" que requieren ser abordados por el maestro directamente con los estudiantes para que puedan continuar con su aprendizaje, tal como lo expresa Lucero:

"Se efectúa una videoconferencia breve, puesto que los alumnos ya estuvieron toda la mañana sentados frente al televisor: se ha dividido al grupo en dos equipos de trabajo de once integrantes... para llevar a cabo las sesiones en línea, no fomenta la convivencia, sin embargo, cuando se conectaba el grueso del grupo a la sesión, no podía visualizarlos a todos en cámara, había exceso de ruido que interrumpía la sesión y en general se presentaban más problemas de conexión entre más participantes había presentes. En promedio los alumnos que se conectan regularmente son 16 ".

Los docentes participantes consideran necesarias sesiones sincrónicas, pero debido a que sus alumnos dependen de un adulto para la conexión y asesoría durante la reunión, deben adaptarse a la disponibilidad de tiempo de los padres de familia, lo que implica una gran disposición de su tiempo incluso, fuera del horario escolar. Sara así lo entiende:

"Debido a que las madres y padres de familia tienen horarios diversos de trabajo, la clase sincrónica se lleva a cabo solo los miércoles; tiene dos horarios, de 4:30 pm a 5:30 pm y de $7.30 \mathrm{pm}$ a 8:30 pm".

García Aretio (2017) considera que una de las características de la educación a distancia es la separación casi permanente del profesor y el alumno en el espacio y en el tiempo, con la salvedad de que, en esta última variable, puede producirse también las interacciones sincrónicas, tal como lo realizan los maestros que participan en el presente estudio.

Uno de los aspectos que más se ha cuestionado a la educación a distancia, es la calidad de ésta. Sin embargo, puede reconocerse como rasgo de calidad la posibilidad de acceder a materiales interactivos, la disponibilidad de aprendizajes sincrónicos que permiten niveles de interacción en tiempo real con mayor capacidad de poder formular preguntas y respuestas inmediatas, tal como lo han implementado los docentes participantes (Rama y Cevallos, 2016). 
De acuerdo con estos autores, la calidad derivada de la interacción de medios visuales, auditivos y escritos, así como desde la interacción y la experimentación, hace que aumenten la retención y los aprendizajes del alumnado.

Al inicio del Ciclo Escolar 2020-2021, y ante el necesario aislamiento social que llevó a la continuidad de clases a través de un sistema a distancia, esta situación desató en los docentes un oleaje de cuestionamientos personales, sociales e institucionales respecto a los diversos métodos, estrategias, técnicas, herramientas, recursos, entre otros, que debían implementar para dar seguimiento a los contenidos de aprendizaje de sus estudiantes. Numerosas dudas y comentarios se compartieron en los grupos conformados por los docentes, quienes, preocupados, esperaban la respuesta que les permitiera continuar de manera exitosa su labor. ¿Qué enseñar? ¿cómo enseñar? ¿cómo evaluar? fueron la constante entre los docentes. Entre lo puesto en práctica se mencionó: elaboración de cuadernillos de hojas impresas con ejercicios de repaso, grupos de WhatsApp y Facebook, videollamadas, fotocopias, libros de texto, llamadas telefónicas, comunicación vía correos electrónicos, uso de diversas plataformas como Microsoft Teams y Google Classroom, y, por supuesto, seguimiento del programa "Aprende en Casa II" (NEXOS, 2020).

Los docentes que participan en este estudio comparten algunas de sus estrategias:

"Se acordó tener clases en línea durante tres días a la semana, un día a la semana trabajar con la programación de "Aprende en Casa II", y otro día trabajar con nuestro foro de lectores y escritores" (Carlos).

"La planeación semanal se centra en los contenidos de "Aprende en Casa" y los Libros de Texto Gratui- tos. A petición de los padres de familia y por disposición de las autoridades educativas, los alumnos basan sus actividades en la programación de TV y suben sus productos a la Plataforma Google Classroom" (Lucero).

Una gran ventaja del programa "Aprende en Casa II" es que los contenidos se encuentran vinculados al programa de educación primaria y a los libros de texto, de tal forma que los niños al seguir el desarrollo del programa pueden complementar el aprendizaje de los diversos temas, con las tareas y actividades didácticas incluidas en sus libros (Comisión Nacional de Libros de Texto Gratuito, 2020).

Para acceder al programa "Aprende en Casa II" es necesario contar con televisión, cuya señal se transmite por televisión abierta en diversos horarios y con una mayor amplitud para una mejor recepción. La maestra Minerva lo confirma:

"Más de la mitad de los padres de familia indicaban que sí contaban con televisión, por lo que se inició trabajando con la programación de "Aprende en Casa"”.

Los docentes refieren que han recibido la indicación de sus supervisores de trabajar de manera exclusiva con las actividades del programa "Aprende en Casa II". Sus planeaciones deben referirse al contenido de la programación.

Entre los retos que se presentan en los hogares para dar continuidad a las actividades escolares aun siguiendo el programa, es que en algunas viviendas se cuenta con una sola televisión, y en ocasiones, son varios niños de edad escolar que requieren visualizar la transmisión, lo que dificulta la continuidad 
de las actividades por parte de los estudiantes. Sumado a lo anterior, existen familias en las que ambos padres son trabajadores y deben cumplir con su jornada laboral, algo que dificulta el monitoreo del avance, así como la atención y seguimiento a las tareas de sus hijos pequeños; en palabras de Minerva:

"Después, se nos dio la indicación (por parte de supervisión) que teníamos que seguir trabajando con el programa “Aprende en Casa II" como principal elemento en nuestras planeaciones, sin embargo, gracias a la comunicación que tengo con los padres de familia, me comentaban que en ocasiones se les hacía muy difícil poder poner a sus hijos a ver la programación, pues a pesar de los horarios flexibles, la mayoría trabajaba. Por lo que se llegó al acuerdo de trabajar con recursos extras, los cuales me han funcionado hasta el momento".

El modelo educativo que prevalece en México durante este periodo de pandemia es la educación virtual, que surge con base en el programa "Aprende en Casa II".

Los docentes se han visto en la necesidad de realizar ajustes a su práctica y a sus planeaciones, incluyendo el empleo de otros recursos como los ya mencionados anteriormente, pues facilitan a los padres de familia dar continuidad a las actividades de sus hijos.

\section{Estrategias de enseñanza- aprendizaje en relación con la lectura y escritura bajo las condiciones de cuarentena que experimentamos}

El tema de la enseñanza de la lectura y escritura ha sido un reto para todos. Los maestros, niños y padres de familia han experimentado diversos sentimientos de confusión, incertidumbre y frustración, entre otros. Entre las estrategias para la enseñanza de la lectura y escritura se mencionan:

"Los días lunes, miércoles y jueves, trabajan... actividades de lectura y escritura, así mismo fortalecemos los contenidos que se trabajan durante estos días para retroalimentar y reforzar aprendizajes, como competencias comunicativas de lectura, escritura, oralidad, cálculo mental y habilidad emocional. Los días martes, trabajan de forma autónoma; en este día procuran desarrollar actividades lúdicas relacionadas a la lectura y escritura, asimismo se envían hojas de trabajo que permiten continuar con los alumnos en aspectos comunicativos y de práctica. En este día los estudiantes se conectan de forma individual o en binas; el propósito de esta comunicación es llevar una conexión, supervisión y tutoría personalizada con cada uno de los estudiantes" (Carlos).

Como puede apreciarse, los docentes han logrado dar seguimiento a la enseñanza de la lectura y escritura a través de diversas actividades. Entre las que mencionan son: foros de lectura y escritura, sopa de letras, memoramas, lotería, crucigramas en hojas de trabajo, creación de cuentos y atención personalizada en sesiones sincrónicas para supervisar avances en lectura y escritura, como lo dicen Carlos y Juana respectivamente:

"El foro de lectores y escritores se aplica los viernes, se convoca tres grupos de alumnos, durante una hora cada grupo, se comparten experiencias y vivencias de lectura y escritura".

"En la mayoría de las materias principalmente español los niños leen y escriben textos cortos, una de las dinámicas que más me gusta trabajar son la creación de cuentos inventados por ellos" 
Además de lo anterior, la maestra Juana da un seguimiento personalizado a los estudiantes que desde su percepción tienen un nivel más bajo de lectoescritura.

"Para los estudiantes que se encuentran con un nivel más bajo de lectoescritura se trabajan actividades con menor dificultad, pero también, atención personalizada mediante WhatsApp y con apoyo de los padres de familia" (Juana).

En relación con la enseñanza de la lectura, los maestros han llevado actividades diversas, y se han apoyado en los libros de texto, en este caso del libro de lecturas que la SEP hecho llegar a los niños de nivel primaria, como es la experiencia de Lucero:

"Dentro del trabajo que se sube en la plataforma Google Classroom se incluyen actividades de lectura y escritura. Se está trabajando con un libro de actividades que complementa al libro de Texto Gratuito de Español Lecturas: se asigna la lectura correspondiente del libro de texto y se sube una hoja de trabajo con tarea".

"Desde el mes de octubre se implementó un Programa de Lectura: se les da a elegir entre dos títulos de libros juveniles para que seleccionen el que más les guste. Tienen un mes para leerlo (se sube el libro en versión digital a Classroom y se envía por correo). El último viernes del mes deben subir un reporte de lectura escrito a mano (para evitar el fenómeno copy/paste en el ordenador) que incluye: autor, de qué trata el libro, su parte favorita, lo que les gustó menos, qué aprendieron del libro, qué cambiarían y su opinión sobre el libro. Este mes están leyendo "Clemencia" de Ignacio Manuel Altamirano".

Para la maestra Lucero, es necesario que los niños elaboren reportes del libro que leyeron; llama la atención de la necesidad de que este se realice "a mano" para evitar que las producciones sean tomadas de alguna página de internet de la que puedan copiar y pegar la información que se solicita. Se evidencia entonces el empleo de la escritura "a mano" como estrategia de aprendizaje.

Otro de los retos a los que se han enfrentado los maestros de primaria es la enseñanza de la escritura, como lo dicen Lucero y Nicolás:

\begin{abstract}
"De la asignatura de español también se han pedido producciones por escrito: una guía de examen, su autobiografía y la biografía de un compañero de clase, un reportaje sobre San Luis Potosí y un cuento de terror. Actualmente están trabajando con un instructivo".
\end{abstract}

"En cuanto a la escritura pido que los papás les hagan dictado, también hay actividades permanentes de caligrafía martes y jueves, mando material impreso, hago video llamadas una vez a la semana para dictarles personalmente y escuchar sus lecturas".

Estos textos deben ser enviados al docente a través del grupo de WhatsApp o subirlos a la plataforma que se haya elegido.

\section{Conclusiones}

Sin duda, la pandemia nos ha tomado por sorpresa. Pronto nos dimos cuenta del desafío que teníamos al intentar continuar con las labores académicas. En el caso de los docentes que participan, expresan que mantener la comunicación con los padres de familia es un reto. La falta de ingresos económicos en algunos casos, así como la dificultad para acceder a señal de internet complejiza el mantenimiento de la comunicación con los padres de familia, y por lo tanto, el seguimiento de los estudiantes. 
Para continuar las actividades educativas, los docentes han considerado los recursos tecnológicos que existen en los hogares y el acceso a internet, pero los horarios de los padres ha sido un elemento fundamental en la organización de las actividades. El uso de grupos de WhatsApp, plataformas virtuales y sesiones sincrónicas han sido parte de las estrategias que emplean los docentes para dar seguimiento a los estudiantes.

Respecto a las estrategias de enseñanza-aprendizaje en relación con la lectura y escritura, los docentes mencionan que las actividades que han puesto en práctica son los foros de lectura y escritura, juegos, sopa de letras, memoramas, lotería, crucigramas en hojas de trabajo, creación de cuentos y sesiones sincrónicas para supervisar avances en lectura y escritura. Respecto al seguimiento de la lectura, se invita a los niños a elegir textos y realizar un reporte de lectura; en cuanto a la escritura, los niños elaboran diversos textos como su autobiografía, la biografía de un compañero, reportaje, cuentos e instructivos que deben subir a la plataforma o enviar evidencia de esta producción. Sin embargo, queda pendiente estudiar los resultados de estas acciones en el aprendizaje de los alumnos.

Conocer las necesidades de estudiantes y docentes en este periodo de contingencia es una necesidad urgente. Analizar la práctica docente bajo las condiciones de pandemia que experimentamos es un gran reto, al igual que conocer con precisión las acciones de enseñanza en la modalidad virtual que los docentes han diseñado para propiciar en los alumnos aprendizajes significativos, es una tarea compleja que requiere de una reflexión amplia y profunda. Además, y no menos importante, dentro del aula se tejen relaciones entre el estudiante y el profesor que impactan en el proceso de enseñanza-aprendizaje, y que ahora, se han transformado profundamente en la educación virtual.

Ha quedado pendiente revisar la valoración de los aprendizajes de los estudiantes en el modelo educativo que permanece en el país. ¿Cómo se lleva a cabo este proceso?, ¿qué instrumentos se han empleado?, ¿qué aspectos se consideran relevantes y necesarios de evaluación? Interrogantes que abren una nueva línea de investigación. 


\section{Referencias}

Balmaceda, J. (2020). ¿Enseñar y Aprender en tiempos de pandemia? En Beltramino, L. (Comp.) Aprendizajes y prácticas educativas en las actuales condiciones de época: COVID-19. Facultad de Filosofía y Humanidades. Córdova. Universidad Nacional de Córdoba. https://drive.google.com/ file/d/1VUsF5hs6kcnPnwz8a6EwDTlWwyLSDQNa/view

Banco Interamericano de Desarrollo (2020). Respuestas al COVID-19 desde la ciencia, la innovación $y$ el desarrollo productivo. https://publications. iadb.org/es/respuestas-al-covid-19-desdela-ciencia-la-innovacion-y-el-desarrollo-productivo

Barrón, M. (2020). La educación en línea. Transiciones y disrupciones. En Casanova, H. (Coord.), Educación y pandemia: una visión académica (pp. 66-74). Ciudad de México: Universidad Nacional Autónoma de México, Instituto de Investigaciones sobre la Universidad y la Educación.

Comisión Nacional de Libros de Texto Gratuito (2020). Aprende en casa con los libros de texto. [Comunicado de prensa]. https://www.gob.mx/conaliteg/prensa/aprende-en-casa-con-los-librosde-texto?idiom $=$ es

Expansión política. (2021). México confirma 1,065 muertes por COVID-19 en el último día. Revista digital. https://politica.expansion.mx/mexico/2021/01/05/ mexico-confirma-1-065-muertes-por-covid-19-en-elultimo-dia
García Aretio, L. (2017). Educación a distancia y virtual: calidad, disrupción, aprendizajes adaptativo y móvil. RIED. Revista Iberoamericana de Educación a Distancia, v.20, n.2, pp. 9-25 . https://doi. org/10.5944/ried.20.2.18737

García, B., Loredo, J. y Carranza, G. (2008). Análisis de la práctica educativa de los docentes: pensamiento, interacción y reflexión. Revista Electrónica de Investigación Educativa, v. 10, pp. 1-15. http://redie.uabc.mx/NumEsp1/contenido-garcialoredocarranza.html

González, G. (2005). Origen y desarrollo de la educación a distancia en México. Encuentro Internacional de Educación Superior. https://www.yumpu. com/es/document/read/6500669/origen-y-desarrollo-de-la-educacion-a-distancia-en-mexico

Huesca, E. (2015) La conectividad, la nube y las transiciones tecnológicas. En Zubieta, J. y Rama, C., (Coord.) La educación a distancia en México. Una nueva realidad universitaria. (pp. 229-241). México: Virtual Educa. Observatorio de la educación virtual.

INEGI (2018). Encuesta Nacional sobre Disponibilidad y Uso de TIC en Hogares. ENDUTIH. https:// www.inegi.org.mx/programas/dutih/2018/

INFOBAE. (2021). Mapa del coronavirus en México 6 de enero: más de 1,000 defunciones y 11,000 contagios en un día. https://www.infobae.com/ america/mexico/2021/01/06/mapa-del-coronavirus-en-mexico-6-de-enero-mas-de-1000-defunciones-y-11000-contagios-en-un-dia/ 
Martínez, C. (2008). La educación a distancia: sus características y necesidad en la educación actual. Educación, v. 33, n. 33, pp. 7-27. http://revistas.pucp.edu.pe/index.php/educacion/article/ view/1532

Mendoza-Castillo, L. (2020). Lo que la pandemia nos enseñó sobre la educación a distancia. Revista Latinoamericana de Estudios Educativos, v. 50, n. ESPECIAL, pp. 343-352. https://doi.org/10.48102/ rlee.2020.50.ESPECIAL.119

Navarrete, Z. y Manzanilla, H., (2017). Panorama de la educación a distancia en México. Revista Latinoamericana de Estudios Educativos, v.13, n.1, pp. 65-82. http://vip.ucaldas.edu.co/latinoamericana/downloads/Latinoamericana13(1)_4.pdf

NEXOS. (2020). Distancia por tiempos. Blog de educación. https://educacion.nexos.com.mx/losmaestros-frente-al-covid-19/

Peters, O. (2002). La educación a distancia en transición. Nuevas tendencias y retos. Universidad de Guadalajara. https://riudg.udg.mx/bitstream/20.500.12104/73591/1/BSUV00011.pdf

Ralón, L., Vieta, M. y de Prada, M. L. V. (2004). (De) formación en línea: acerca de las desventajas de la educación virtual. Comunicar, n. 22, v. 11, pp. 171176. https://www.revistacomunicar.com/ojs/ index.php/comunicar/article/view/C22-2004-26
Rama, C. y Cevallos, M. (2016). Nuevas dinámicas de la regionalización universitaria en América Latina. Magis, Revista Internacional de Investigación en Educación, 8, (17), 99-134. https://doi. org/10.11144/Javeriana.m8-17.ndru

Rodríguez, G., Gil, J. y García, E. (2006). Metodología de la investigación cualitativa. Ediciones Aljibe.

Secretaría de Educación Pública (SEP) (2020a). Boletín No. 99 Difunde SEP horarios del programa Aprende en Casa. Blog de la Secretaría de Educación Pública. https://www.gob.mx/sep/articulos/ boletin-no-99-difunde-sep-horarios-del-programa-aprende-en-casa

Secretaría de Educación Pública (SEP) (2020b). Boletín No. 113 Programa Aprende en Casa diseñado $y$ evaluado por maestros especialistas en educación básica: SEP. https://www.gob.mx/sep/articulos/ boletin-no-113-programa-aprende-en-casa-disenado-y-evaluado-por-maestros-especialistas-en-educacion-basica-sep?idiom $=\mathrm{es}$

Secretaría de Educación Pública (SEP) (2020c). Boletín No. 80. Fortalece SEP programa Aprende en Casa mediante sitio web especializado en educación básica. SEP. https://www.gob.mx/ sep/es/articulos/boletin-no-80-fortalece-sepprograma-aprende-en-casa-mediante-sitio-webespecializado-en-educacion-basica?idiom=es 
Secretaría de Educación Pública (SEP) (2020d). Boletín No. 245 Beneficia Regreso a Clases. Aprende en Casa II a 30 millones de niñas niños, adolescentes y jóvenes. Blog de la Secretaría de Educación Pública. https://www.gob.mx/sep/articulos/boletin-no245-beneficia-regreso-a-clases-aprende-en-casaii-a-30-millones-de-ninas-ninos-adolescentes-yjovenes-sep?idiom=es

Secretaría de Educación Pública (SEP). Acuerdo número 02/03/20 (2020) por el que se suspenden las clases en las escuelas de educación preescolar, primaria, secundaria, normal y demás para la formación de maestros de educación básica del Sistema Educativo Nacional, así como aquellas de los tipos medio superior y superior dependientes de la Secretaría de Educación Pública. Diario Oficial de la Federación.
Secretaría de Educación Pública. (SEP) (2020). Acuerdo número 02/03/20 (2020). Por el que se suspenden las clases en las escuelas de educación preescolar, primaria, secundaria, normal y demás para la formación de maestros de educación básica del Sistema Educativo Nacional, así como aquellas de los tipos medio superior y superior dependientes de la Secretaría de Educación Pública. Diario Oficial de la Federación. Secretaría de Educación Pública.

Tintaya, E. (2003). Desafíos y fundamentos de educación virtual. Material de enseñanza. Universidad Mayor San Andrés, Ciencias de la Educación. https://cursa.ihmc.us/rid=1367905953779_1796 184220_47076/Desafios_y_Fundamentos_de_ Educacion_Virtual.pdf

Zabala, A. (1995). La práctica educativa. Ed. Graó. 